\title{
Complex and Diverse Morphologies Can Develop from a Minimal Genomic Model
}

\author{
Jose David Fernández \\ Research Group in \\ Biomimetics, Department of \\ Computer Science, University \\ of Málaga, Severo Ochoa 4, \\ 29590 Málaga, Spain \\ josedavid@geb.uma.es
}

\author{
Francisco J. Vico \\ Research Group in \\ Biomimetics, Department of \\ Computer Science, University \\ of Málaga, Severo Ochoa 4, \\ 29590 Málaga, Spain \\ fjv@geb.uma.es
}

\author{
René Doursat \\ Research Group in \\ Biomimetics, Department of \\ Computer Science, University \\ of Málaga, Severo Ochoa 4, \\ 29590 Málaga, Spain \\ doursat@yahoo.com
}

\begin{abstract}
While development plays a critical role in the emergence of diversity, its mechanical and chemical actions are considered to be inextricably correlated with genetic control. Since in most extant species the complex growth from zygote to adult organism is orchestrated by a complex gene regulatory network (GRN), the prevalent view is that the evolution of diverse morphologies must result from the evolution of diverse GRN topologies. By contrast, this work focuses on the unique effect of developmental processes through an abstract model of self-regulated structure without genetic regulation - only modulation of initial conditions. Here, morphologies are generated by a simple evolutionary algorithm searching for the longest instances of unfolding dynamics based on tensegrity graphs. The usual regulatory function of the genome is taken over by physical constraints in the graphs, making morphological diversity a pure product of structural complexification. By highlighting the potential of structural development, our model is relevant to both "structuralist" biological models and bio-inspired systems engineering.
\end{abstract}

\section{Categories and Subject Descriptors}

J.3 [Life and medical sciences]: Biology and genetics; I.2.8 [Artificial Intelligence]: Problem Solving, Control Methods, and Search-Heuristic methods; G.1.6 [Numerical Analysis]: Optimization-Global optimization

\section{General Terms}

Algorithms, Design

\section{Keywords}

computational biology, evolutionary development, morphogenesis, indirect encoding, tensegrity

Permission to make digital or hard copies of all or part of this work for personal or classroom use is granted without fee provided that copies are not made or distributed for profit or commercial advantage and that copies bear this notice and the full citation on the first page. To copy otherwise, to republish, to post on servers or to redistribute to lists, requires prior specific permission and/or a fee.

GECCO'12, July 7-11, 2012, Philadelphia, Pennsylvania, USA.

Copyright 2012 ACM 978-1-4503-1177-9/12/07 ...\$10.00.

\section{INTRODUCTION}

The phenotype of a multicellular organism is generated by a complex developmental process, which transforms a single fertilized cell, the zygote, into a living architecture composed of millions to trillions of cells. This process is a fascinating self-made choreography of precisely timed events, during which cells in the growing organism spontaneously divide, modify their physicochemical properties, and arrange into layers and tissues, themselves folding into increasingly complicated shapes to form organs and appendages [9]. The recent discipline of evolutionary developmental biology, or "evo-devo", studies development from an evolutionary perspective (and vice-versa). It examines how phenotypic variation arises from the interplay between the physics of growth and both genetic and environmental variations. Evo-devo takes the viewpoint that morphological trends in evolution are biased to a large extent by developmental mechanisms [1], re-interpreting the traditional but simpler concept of "phenotypic plasticity" [7]. It also applies to some unicellular organisms, albeit at the molecular scale [11].

In this context, genetic regulatory networks (GRNs) are emerging as a unifying operating concept [5]. A GRN model postulates that the genome can be described as a vast and complex network of interacting genes, promoting and repressing each other's expression through the intermediation of produced proteins that bind to specific regulatory sites on the DNA. Abstracting out the convoluted pathways and feedback loops between genes, RNA, proteins and metabolites, the state of a GRN can be formalized as a set of "transcription levels" on each gene-node - in the simplest of cases, a binary graph. It can be conceptualized as a dynamical system that follows different trajectories in state space, typically landing on fixed-point or limit-cycle attractors. Each attractor-subset of genes can then be interpreted as a cell type with precise behavior and properties [19]. Since the discovery of the homeobox genetic toolkit, development is construed as a complex process tightly modulated by an underlying GRN model: large-scale, coordinated changes in the phenotype are a more or less direct consequence of small, localized changes in the regulation of the developmental process [7], be they genetic [36] or environmental [38] changes.

\subsection{Reinstating Development}

However, while the detailed knowledge of genomic sequences enables scientists to pinpoint where and when different genes are expressed in the embryo, it is insufficient 
to understand how the organism builds itself [27]. Regulating and regulated genes of a GRN are separated in reality by the major gap of development, which comprises cellular biomechanics and chemical signaling. To fill this gap, evo-devo researchers describe developmental processes as a coupling between GRNs and the physical properties of the organisms $[7,9]$. The aim of the present work is to focus on the latter, specifically the ability of tensegrity structures (see Section 2.1) to generate a wide diversity of forms.

Complex morphologies can be generated by developmental processes. Mechanical cues (strain, compression, bending forces, etc.) play a key role in development by modulating cell differentiation, influencing the direction of growth, and, of special interest here, deforming tissues [13]. Even late-stage (post-embryogenesis) events, such as the folding of the mammalian gut, are primarily determined by mechanical dynamics [33]. Geometrically, the typical embryogenesis of a triploblastic animal consists of a set of complex and precisely timed developmental steps, which dynamically change the physical properties of the cells, the surrounding extracellular matrix and the differential adhesiveness among cells and between cells and other substrates [9].

At a mechanical level, several types of morphogenetic processes can be distinguished, such as cell sheets growing in a given direction, or cells detaching from these sheets and migrating to create new groups. Some of the most common morphogenetic processes include "invagination", in which a sheet of cells folds inward, possibly resulting in the folded cells detaching to form another sheet (e.g., the neural tube), and "branching", in which a tube recursively branches off into a fractal-like pattern (e.g., lung organogenesis [16] or vasculogenesis [21]).

\subsection{Relegating Genetic Control}

In sum, for evo-devo, biological development consists of a precise spatio-temporal cascade of developmental stages in which morphodynamic physical processes are continually regulated by the genome at multiple levels [27]. Rewinding the course of evolution, however, one can make the hypothesis that in the beginnings of multicellular life the morphologies of organisms might have been essentially generated by physical processes and their environmental conditions, while it is only later that gene regulation had stepped in and taken control [29]. In this paradigm, morphologies made their first appearance as a contingent, but deterministic, result of physicochemical constraints, after which new layers of regulation mechanisms encoded in the DNA helped stabilize these pre-existing developmental processes and expose them to genetic variation and natural selection.

The present study broadly belongs to artificial development (AD), an emerging field at the crossroads of computational biology, artificial life and evolutionary computation that studies computational models of development toward various scientific and engineering applications [35]. Typically, the use of evolutionary methods to conduct a parameter search or stochastic optimization becomes especially difficult when the individuals are complex systems, i.e. made of a large number of interacting components, because of their high dimensionality (number of degrees of freedom). As a new avenue of bio-inspiration, $\mathrm{AD}$ therefore offers interesting approaches to encode complex problems in a compact way, via a developmental or generative stage. AD genomes are cases of "indirect" encodings whose size is significantly smaller than "direct" encodings [35], thus greatly improving the efficiency of evolutionary algorithms (despite the added developmental calculation).

However, the introduction of a developmental stage adds a new layer of complexity in some cases. In most AD models, indirect encoding remains a complex interaction between the genotype (often a GRN) and the developing phenotype, for example in generative architectures based on L-system grammars [14], Framsticks robots using genetic programming [20] or path-follower creatures produced by graph rewriting [22]. Some researchers have investigated more complex and biologically realistic models of developing phenotypes with the intention of minimizing the size and complexity of the genotype even further to make it more amenable to evolutionary methods [8]. In this context, our study represents an exploration of how small the genetic component of an AD model can become, while also keeping structural principles simple and still enabling an evolutionary search to solve a problem - in this case, give rise to a diversity of morphologies. To this aim, we use tensegrity structures, as explained in the next section.

\section{MECHANICAL MODEL}

\subsection{The Potential of Tensegrity}

Historically, many definitions of tensegrity structures have been proposed [18]. Most of them are variations on the original definition by Snelson [34] and Fuller [10], respectively the creator and namer of this concept (from a contraction of "tensional integrity"). The most generic definition of tensegrity involves a structure composed of compression-bearing rigid elements (rods) isolated from each other and connected by a network of tensioned cables (springs), such that the sum of forces acting on each vertex is null [25] and the structure is maintained in mechanical equilibrium. The balance of strains and compressive forces sustained by each tensegrity element is called the self-stress of the structure and characterizes the high structural resilience of the whole. Combined with an exceptionally high rigidity-to-mass ratio, all these properties make tensegrity an object of active study with numerous applications in architecture, civil and mechanical engineering [25].

The last decade has also seen many applications of tensegrity structures to robotics. They comprise the design of control systems in walking robots by analytical [12] or evolutionary [31] methods, the optimization of the dynamical response of articulated wing frameworks [2], the reliance on large-scale couplings and nonlinear dynamics in tensegrity to achieve locomotion with minimal control [32], and the design of ballistic structures able to withstand landing impacts by minimizing the effects of compression shocks [23].

The concept of tensegrity has also been extensively used in biology, notably by Ingber at the molecular and cellular levels [15]. His proposal that the cytoskeleton can be modeled as a dynamic tensegrity structure has led to a new explanation of several properties of cells and tissues in terms of tensegrity properties, in particular:

- the exceptional resilience of the cytoskeleton [3] as an effect of uniform load distribution in tensegrity structures;

- the complex mechanical response of the cell $[15,4]$ to deformation and substrate-dependent shape change; 


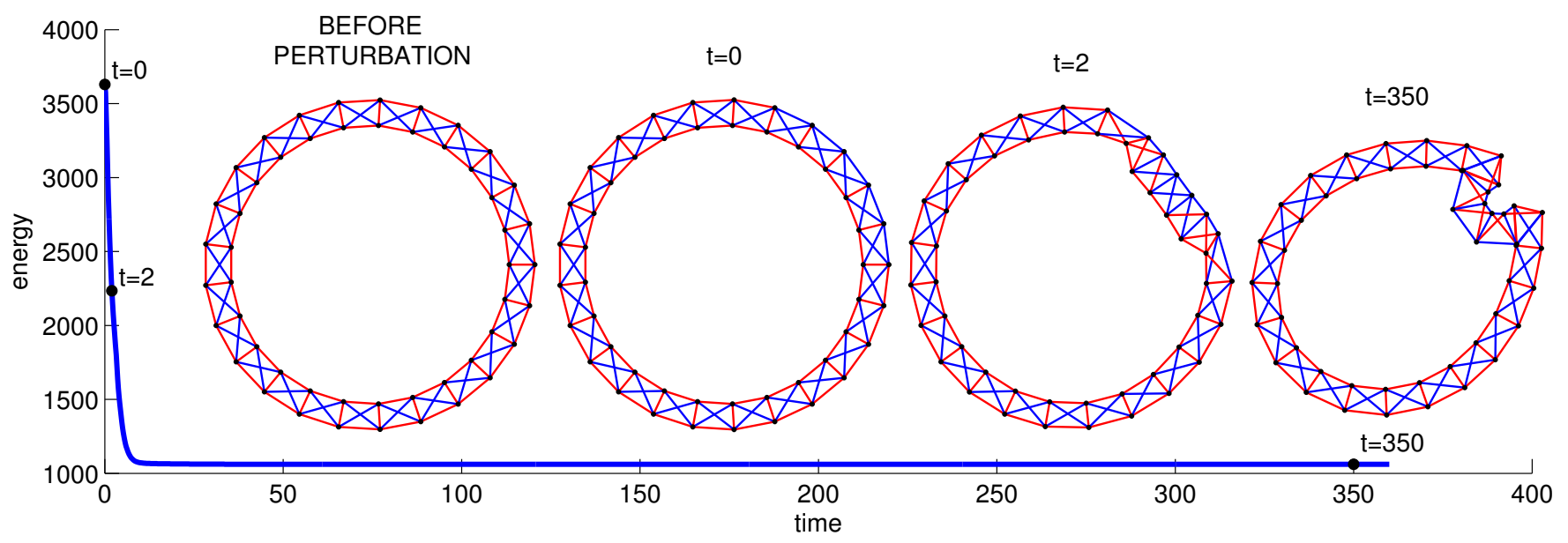

Figure 1: A 2D circular sheet of cells modeled as an elastic tensegrity structure (see text). A morphogenetic event (invagination) is triggered by shortening the peripheral elastic links of a group of neighboring cells [30]. During the subsequent relaxation process, the structure reaches a new equilibrium state. Three snapshots are shown: start of simulation $(t=0)$, midway folding $(t=2)$, and final state $(t=350)$. Blue plot: total energy as a function of time (notice the sharp drop).

- the conversion of mechanical signals into biochemical responses, or "mechanotransduction" relying on force distribution throughout the cytoskeleton [17].

These properties have important consequences on development, too. As sheets of cells expand and cells assemble in specific locations, they become subject to forces and strains that provide mechanical cues, which in turn eventually affect the timing of the growth stages. The tensegrity properties of the cytoskeleton are critical in supporting this whole process [24].

\subsection{A Circular Sheet of Cells}

In this section, we describe a model of development with minimal genetic control based on tensegrity structures. Since the elements of a tensegrity structure store potential energy, the structure can find itself in an unstable state. If the balance of forces is perturbed beyond a certain threshold, whether by shape deformation or modification of the stress properties of the elements, the structure will "snap" to accommodate these new constraints. It will then settle again into a lower-energy state possibly characterized by a very different shape, dissipating potential energy in the process.

Our work exploits this propensity to "catastrophic" state change or "phase transition" exhibited by tensegrity structures under certain stress conditions. It is inspired by a simple 2D developmental model of invagination [26, 30], in which a circular sheet of cells is modeled as a closed chain of cellular cytoskeletons. Each cell's cytoskeleton consists of six overdamped elastic edges: four external edges arranged in a quadrilateral plus their two diagonals. These quadrilaterals are then merged together to form a circular elastic network representing a closed sheet of cells (Figure 1, first structure). As a whole, the sheet constitutes a self-stressed tensegrity structure potentially capable of settling into multiple configurations of compressed and strained elements. For example, to provoke an invagination process in one region of the cell sheet, it is sufficient to shorten the resting length of the outer elastic links in that region (Figure 1, second to fourth structures). This shows that a very simple and localized genetic signal controlling only a few cytoskeletal features is able to induce a dramatic phenotypic change.

We adopt a similar 2D structure (see Figure 2b) as a starting point for the present evolutionary study, leaving aside its origins as a cell sheet model. Instead, we construe it from now on as an abstract model of morphogenetic processes, by which shapes can undergo a complex but well-ordered series of morphological transformations (keeping in mind that some transformations might not be topologically possible in more realistic 3D structures). We show that elastic tensegrity networks are able to rearrange themselves under minimal genetic control and give rise to a diverse array of morphologies. Such behavior makes these structures good candidates to represent the physical substrate of complex development, whether in biological modeling or in bio-inspired engineering.

\section{EVOLUTIONARY DEVELOPMENT}

An evolutionary algorithm is used to explore the parameter space of morphogenetic tensegrity processes. The information comprised in each gene of an individual is interpreted as a local or global perturbation to be applied to its structure at a given developmental stage - here, the initial circular sheet common to all individuals. Thus the genome does not represent the layout of the organism, but scheduled sequence of changes in the internal balance of forces. These changes accumulate potential energy in the structure, and new arrangements of cells emerge from their subsequent expression and unfolding. In summary, to evaluate an individual, the initial structure is first modified according to its genome, then goes through a physical relaxation process, which may include one or several abrupt transitions, until it stabilizes into a new shape. The evaluation is the measure of the "quality" of this morphogenetic process, as it will be explained below. 


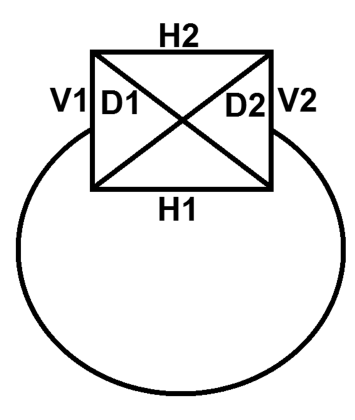

(a)

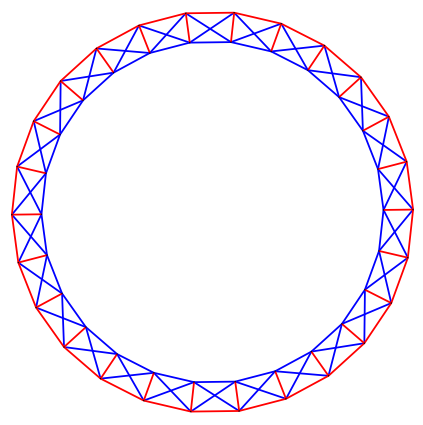

(b)
Figure 2: (a) The six elastic links composing each quadrilateral cell. (b) Circular tensegrity structure of 26 connected cells used as the common initial state of all morphogenetic processes. The $V_{2}$ link of each cell is superimposed on the $V_{1}$ link of its neighbor clockwise. H1's resting length is smaller than its length in the circle, while H2's is longer (see Appendix). Compressed links are red, stretched ones are blue.

\subsection{Individuals}

We denote by $H_{1}, H_{2}, V_{1}, V_{2} D_{1}$, and $D_{2}$ the six types of elastic links of a single quadrilateral cell (Figure 2a), and by $L_{i j}$ an instance of (undirected) link between two vertices $i$ and $j$. The force exerted by $L_{i j}$ on $i$ at any time is given by $\mathbf{F}_{i j}^{e}=-k_{i j}\left(d_{i j}-l_{i j}\right) \mathbf{u}_{i j}$, where $k_{i j}$ is a positive stiffness coefficient, $l_{i j}$ the resting length of link $L_{i j}, d_{i j}$ its current length (absolute values), and $\mathbf{u}_{i j}$ a unit vector oriented from $j$ to $i$. The initial structure is in equilibrium, i.e. $\mathbf{F}_{i}^{e}=$ $\sum_{j} \mathbf{F}_{i j}^{e}=\mathbf{0}$ for all $i$.

Each cell in the initial sheet structure is indexed by an integer $c \in[1,26]$. The genome of a structure is encoded in a list of variable length, where each perturbation element is represented by a quintuple denoted $\left(c_{a}, c_{b}, S, T, x\right)$ with the following definitions:

- $c_{a}$ and $c_{b}$ are two integer indices in the range $[-5,30]$ such that the perturbation is applied only to the cells that verify $c \in\left[\min \left(c_{a}, c_{b}\right), \max \left(c_{a}, c_{b}\right)\right] \cap[1,26]$

- $S$ is a subset of link types $\left\{H_{1}, H_{2}, V_{1}, V_{2}, D_{1}, D_{2}\right\}$ such that, inside each cell $c$, the perturbation is applied only to the categories of links listed in $S$

- $T$ is a rule type symbol from the set $\left\{K, R, K_{f}, R_{f}\right\}$ and $x$ is a real-valued coefficient in $[0,2]$, such that:

- if $T=K$, the link's stiffness $k$ is multiplied by $x$

- if $T=R$, its resting length $l$ is multiplied by $x$

- if $T=K_{f}$, same effect as $K$; in addition, $l$ is also readjusted such that the force value $F^{e}=$ $-k(d-l)$ is unchanged (under the link's current length $d)$

- if $T=R_{f}$, same effect as $R$ using coefficient $x^{\prime}$ instead of $x$ such that: if $x>1$ then $x^{\prime}=x$ for a compressed link $(l>d)$ and $x^{\prime}=1 / x$ for a stretched link $(l<d)$, and conversely for $x<1$; in addition, $k$ is also readjusted such that $F^{e}$ is unchanged.
All perturbations coded in the genome are applied to the initial circular sheet in the order they appear in the list, then the structure is left free to rearrange itself. Note that, while perturbations of types $K_{f}$ and $R_{f}$ leave the balance of forces seemingly unchanged, they significantly alter "undercover" the mechanical response of affected links to subsequent perturbations, thus greatly influencing the developmental process. Finally, after all perturbations have been applied, links of stiffness $k$ lower than a given threshold are discarded because they play no significant role in the dynamics. Likewise, the values of $k$ or $l$ are limited to given maximum values to prevent the system from becoming unstable.

A remark about the computational size of the genome: since $c_{a}, c_{b}, S$ and $T$ can each be represented by one byte, and $x$ is a double-precision floating-point variable, a perturbation can be encoded in just 12 bytes (or even 11, if $S$ and $T$ are crammed into a single byte). Therefore, with typical evolved genomes containing 2 to 5 perturbations, their size ranges from 10 to 25 variables, or 24 to 60 bytes. Thus the "minimality" of the genome in this work can be understood as referring to its small size as well as the simplicity of the mapping.

\subsection{Evaluation}

Denoting the position of vertex $i$ by $\mathbf{P}_{i}$, we can write $d_{i j}=\left\|\mathbf{P}_{i}-\mathbf{P}_{j}\right\|$ and $\mathbf{u}_{i j}=\left(\mathbf{P}_{i}-\mathbf{P}_{j}\right) / d_{i j}$. The equation of motion of $i$ then reads $\ddot{\mathbf{P}}_{i}=\mathbf{F}_{i}^{e}+\mathbf{F}_{i}^{v}$, where $\mathbf{F}_{i}^{e}$ is the sum of elastic forces:

$$
\mathbf{F}_{i}^{e}=-\sum_{j=1}^{N} A_{i j} k_{i j}\left(\left\|\mathbf{P}_{i}-\mathbf{P}_{j}\right\|-l_{i j}\right) \frac{\mathbf{P}_{i}-\mathbf{P}_{j}}{\left\|\mathbf{P}_{i}-\mathbf{P}_{j}\right\|}
$$

$\left(A_{i j}=1\right.$ or 0 denoting the existence or absence of $\left.L_{i j}\right)$ and $\mathbf{F}_{i}^{v}=-\mu \dot{\mathbf{P}}_{i}$ is a linear damping force, with $\mu=0.1$.

After perturbing the circular sheet according to the genome of an individual, the resulting structure is generally unstable and its numerical simulation requires a fourthorder Runge-Kutta integrator with time step $\Delta t=0.01$. We calculate the total energy of the system $E=E_{k}+E_{p}$, where $E_{k}=\frac{1}{2} m \sum_{i}\left\|\dot{\mathbf{P}}_{i}\right\|^{2}$ is the kinetic energy of the vertices and

$$
E_{p}=\frac{1}{2} \sum_{i, j=1}^{N} A_{i j} k_{i j}\left(\left\|\mathbf{P}_{i}-\mathbf{P}_{j}\right\|-l_{i j}\right)^{2}
$$

is the potential energy stored in the elastic links. Typical coefficient values are $m=1$ and $k_{i j}=20$, making $E_{k}$ of the order of $10 \%$ of $E$ in the very first steps of the relaxation, then at best a fraction of $1 \%$ of $E$ on the long run (Figure 3). As $E$ decreases over time, we stop the relaxation process when the rate of energy loss $|\dot{E}|$ drops below a given threshold, meaning that the structure is close to its final stable configuration.

In a simple metastable system such as the invagination process of Figure 1, the relaxation from the initial to the final state was following an exponential-looking energy curve, characterized by a sharp drop before a slow decrease. There, the potential energy was spent in one shot. However, tensegrity structures are also able to exhibit much longer and more complex transformations, consisting of a diverse sequence of developmental stages and qualitative transitions (Figure 3). In order to encourage this diversity, we propose to detect the transitions through the conspicuous accelerations that they produce in the energy loss rate, then use them to count 


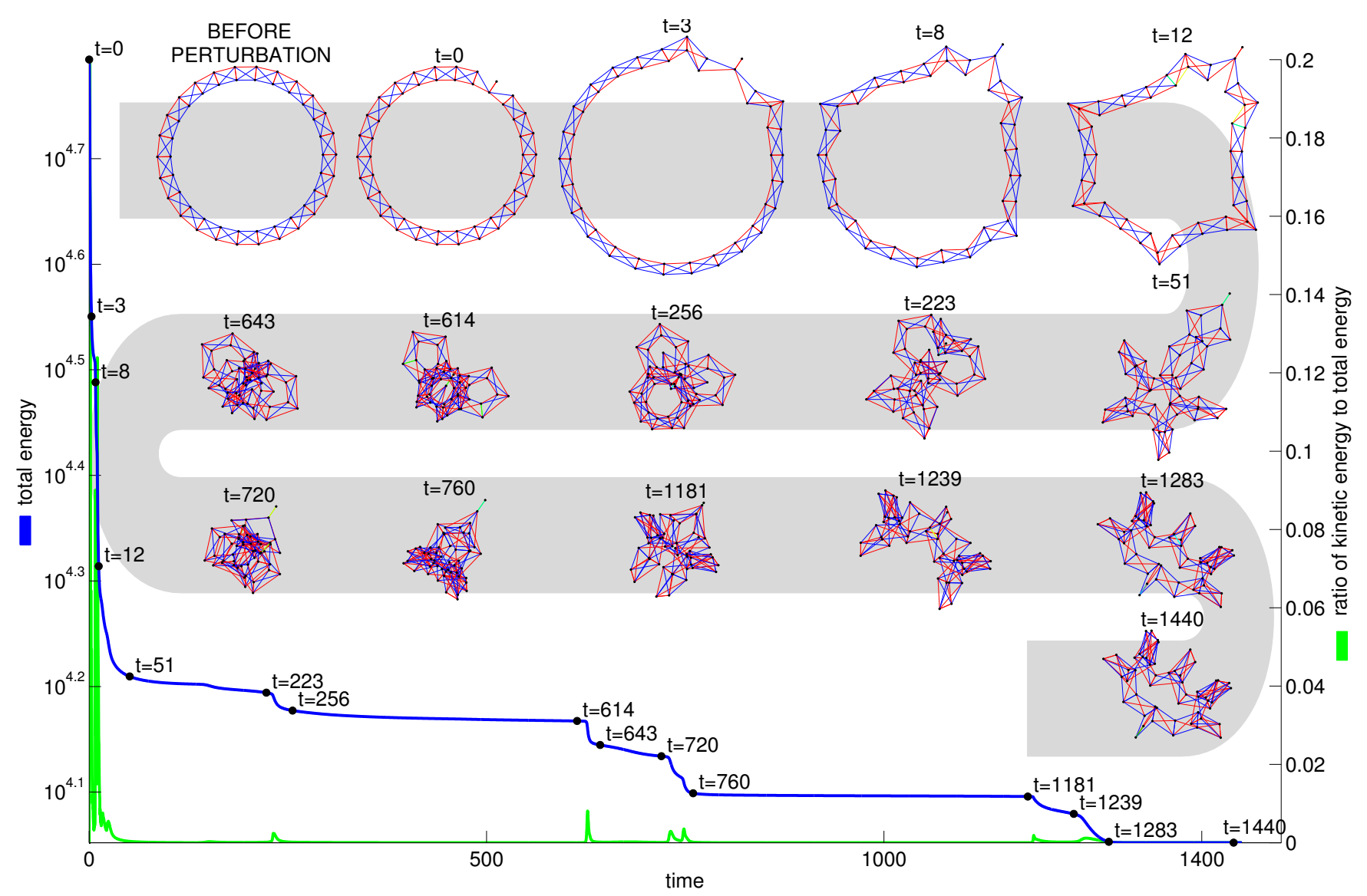

Figure 3: An example of developmental process with minimal genetic control. See the text for details.

the number of developmental steps (energy plateaus), their size and distribution, which will allow us to design a fitness or measure of "quality" of the process.

Staircase steps in the energy curve $E(t)$ appear as peaks and valleys in its first derivative $\dot{E}(t)$. We count only the peaks in energy rate that have a maximal value greater than a given threshold. Let $a_{q}$ be the time of peak $q, b_{q-1}$ and $b_{q}$ the times of the valley flats before and after $a_{q}$, and $d_{q}=E\left(b_{q-1}\right)-E\left(b_{q}\right)$ the energy drop corresponding to peak $q$. Denoting by $\mathbf{D}^{\prime}$ the complete sequence of energy drops in decreasing order of magnitude: $\mathbf{D}^{\prime}=\left(d_{1}^{\prime}>\cdots>d_{n}^{\prime}\right)$, we first discard its largest component $d_{1}^{\prime}$ (in most cases the initial peak: $d_{1}^{\prime}=d_{1}$ ), viewing it as an outlier. Then, we rescale the values in the reduced set with respect to the second maximum $d_{2}^{\prime}$ and define the fitness $f$ as the sum of these values:

$$
f=\sum_{k=2}^{n} \frac{d_{k}^{\prime}}{d_{2}^{\prime}}
$$

Therefore, by attempting to maximize this function, the goal is to favor long and complex developmental processes characterized by an energy profile made of multiple steps of similar size.

\subsection{Selection}

Randomly generated individuals are unlikely to produce complex developmental processes and diverse morphologies. For this reason, we used the following evolutionary protocol:
1. First, 100 individuals are randomly generated and evaluated. This set constitutes the initial population.

2. Then, the following steps are repeated 500 times:

2.1 individuals from the current population are selected through a size- 4 tournament, with elitism, to generate a new population (i.e., the best individual is automatically copied, while the other individuals compete in randomly chosen groups of 4)

2.2 some individuals of the new population are mutated and re-evaluated.

Three types of mutations affecting the list of "perturbation" genes $\left(c_{a}, c_{b}, S, T, x\right)$ are possible:

- delete a randomly chosen gene

- change the value of a randomly chosen component of a randomly chosen gene

- insert a randomly created gene at a randomly chosen location in the gene sequence.

Each mutation is applied to each individual with probability 0.05 . The initial 100 individuals contain exactly two randomly created genes, in such a way that the first perturbation gene is always global (i.e., the interval defined by $c_{a}$ and $c_{b}$ includes $[1,26]$ completely), while the second perturbation gene is always localized to 5 cells or less (i.e., $\left|c_{a}-c_{b}\right| \leq 5$ ). 


\section{EXPERIMENTAL RESULTS}

Through several runs of the above evolutionary algorithm, we found many examples of long processes containing multiple developmental steps. A sizable fraction of these cases were discarded because, for example, the structure would collapse into a small area and make it difficult to visualize a shape. Since the heuristics only relies on an indirect criterion (maximizing $f$ ), some processes did not resemble any sequence of developmental steps. In numerous other examples, however, the structure did not collapse upon itself and even exhibited interesting bilateral symmetry. In symmetric structures, shape creation could be followed visually as different segments of the cell sheet folded in various ways and gave rise to a wide variety of transient and final morphologies. Figure 3 is illustrative of two typical properties of a "successful" developmental process:

- Developmental steps are characterized by periods of smooth changes separated by sudden transformations, during which potential energy is rapidly converted to kinetic energy, then dissipated, causing a drop in total energy. In most cases, these transitions correspond to sudden reversals in the positions of the extremities of one or several elastic links. The plots of Figure 3 show the typical staircase profile of total energy over time (blue line), $E(t)$, and the ratio of kinetic energy (green line), $E_{k}(t) / E(t)$ (drops in the former coinciding with peaks in the latter).

- The structure undergoes dramatic rearrangements. Snapshots of the different stages of development are also shown in Figure 3. The gray path indicates their chronological order. Each snapshot is taken just before or after one of the transition steps characterized by a sudden energy drop (times are indicated on the figures and the corresponding locations on the plot).

In this typical example, two regimes in the dynamics of $E(t)$ can be clearly distinguished:

- An initial phase where most of the energy is dissipated in a fraction of time. This first and intense "snap" corresponds to the setup of the developmental process, modulated by the local and global genomic perturbations that were stored in the structure.

- A much longer regime during which the remaining energy is dissipated in much smaller quantities (the vertical axis in Figure 3 is a logarithmic scale to show these steps better). This "slow" regime corresponds to the developmental process proper and embodies the essential differences between examples.

The initial conditions of this example are configured by the following genome, composed of a global perturbation followed by two local perturbations:

$$
\begin{aligned}
& \left(1,26,\left\{H_{1}\right\}, R, 2.145\right) \\
& \left(1,1,\left\{H_{2}, D_{1}\right\}, K, 0.0\right) \\
& \left(2,2,\left\{H_{2}, D_{2}\right\}, K, 0.0\right)
\end{aligned}
$$

Global perturbations have the effect of introducing a significant amount of potential energy and destabilizing the initial structure. Local perturbations canalize the destabilized structure into an specific developmental path by modulating the location and dynamics of the initial catastrophic events (reversals of elastic links).

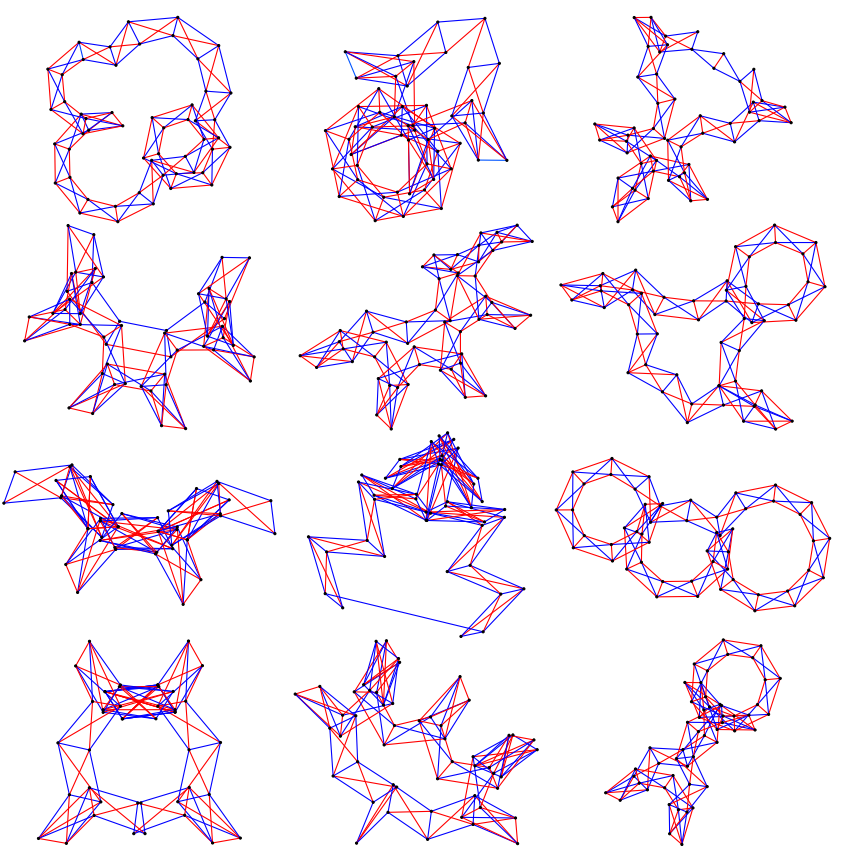

Figure 4: Various examples of final morphologies produced by different developmental processes, exhibiting more or less symmetry. All these morphologies are generated from the same initial structure (Figure 2b) by modulating the perturbations applied to that structure.

Many examples of final, stable morphologies have been evolved. As shown in Figure 4, they strikingly differ from each other, although they all originate from the same initial cell sheet structure through folding.

\section{DISCUSSION}

In this study, we proposed a model of development able to give rise to a diversity of morphologies under minimal genetic control. While it is common to consider that, in most cases, a complex genetic control is necessary to guide an equally complex developmental process, essentially by intervening at bifurcation points to switch on and off distinct, heterogeneous phases (see, e.g., [6]), our model represents an extreme example of self-controlled physical structure. No GRN is needed to guide the unfolding of its dynamics, since the properties of metastable tensegrity modulate themselves the sequence of transformation steps. After perturbation in a few specific points, the structure follows a complex sequence of morphological rearrangements and state transitions before settling down into a final configuration.

This whole process is enabled by the "self-stress" features of the circular sheet used as the initial state of the model. This structure is stable in geometric configurations that correspond to local minima of the energy function (see Section 2.1), and the role of the perturbations specified by an individual's genome is precisely to alter the shape of this energy function. This drives the structure into a developmental path (a trajectory in phase space), where each step is a consequence of the geometric changes created in previous steps, until a new balance of forces is attained. Although our primary goal was to study development as such, this method 
can also be seen as a new way to generate novel tensegrity structures (see Section 2.1 for other methods).

\subsection{Relevance to Biological Modeling}

With respect to evo-devo, we believe that the significance of our model is to cast a new light on the question still little addressed in today's predominant gene-centric view of biology: Can organisms also be the product of complex physicochemical developmental processes not (necessarily or always) controlled by complex underlying genetics? This question was mostly investigated by the "structuralist" school of theoretical biology, which can be traced back to Goethe, D'Arcy Thompson, and Waddington (before or during the advent of genetics and its modern synthesis with evolution). Later, in the genomic era, it was most actively pursued and defended by Kauffman [19] and Goodwin [11] under the banner of self-organization, argued to be a greater force than natural selection in the production of viable diversity.

Each element of our tensegrity graphs influences the stress state of the other elements in convoluted, nonlinear ways, creating the conditions for enhanced developmental effects, while only a small amount of genetic information is sufficient to encode structural perturbations. This can also be seen in unicellular organisms whose complex cytostructure develops from an undifferentiated stage. A classical example is Goodwin's model of Acetabularia [11], a genus of green algae in which the genome does not explicitly code for the branching "bracts" (rings of little leaflike elements) of a growing cell. These bracts are only the indirect result of a set of physical initial conditions, which can be modulated by the genome to produce different morphologies in different species. This represents a real-life example of developmental process primarily based on the physical properties of the developing structure, as in our model.

As seen in Figure 3, one tensegrity individual is characterized by its typical sequence of variation steps, or "signature", of its energy over time. These steps are "catastrophic" geometric events, in the sense of René Thom's theory [37], frequently involving reversals of the elastic links, which cause a self-propagating cascade of dramatic jumps in the balance of forces. In Kauffman's interpretation of Waddington's epigenetic landscapes, cell types are metastable states of the cell's genetic regulatory network [19]. The morphodynamics described in this work could thus be considered a purely physical analog of Kauffman's metaphor, at an abstract level of self-organization. A metastable morphology represents a "transient attractor" in the physical dynamics of the structure, as it visits these attractors before arriving in its final state.

Although our model is not related to any real-world biological system, it represents a proof of concept that complex developmental processes do no always need to be controlled by genetics but can largely self-regulate by relying on the physical properties of growing tissues [28]. In this respect, it lends support to the hypothesis that, in the beginnings of multicellular life, organismal development was under little to no genetic regulation [29], instead being guided by structural constraints.

\subsection{Relevance to Bio-inspired Engineering}

From the point of view of evolutionary computation and systems design, the aims of artificial development are to discover and improve algorithms by leveraging self- organizational processes to code complex solutions with small genomes [8]. Yet, even in this field especially suited to the design of "embodied" agents, a common assumption is that generating architectures complex enough to be capable of spontaneous innovation requires direction from a complex GRN or GRN-like mechanisms distinct from the physical model proper. As mentioned in Section 1.2, many AD models choose to support complexity in the development by a relative complexity in its recipe $[8,14,20,22,6]$.

Our developmental model is purely "ballistic" in the sense that genetic control is limited to providing perturbations to an initial metastable structure. In this respect, it represents a proof of concept that AD-based evolutionary algorithms [35] can encode solutions to form-finding problems in extremely concise ways. Earlier explorations of this concept [8] have used more complex genetic machinery, which we could avoid here by using tensegrity structures.

Naturally, starting from this minimal base, it would be also perfectly possible to extend the model and reintroduce more genetic information. For example, one could allow perturbations to be triggered not only at the beginning of development but at later times, too, through various physical parameters. This would certainly allow for an even more complex and more reactive growth dynamics, better resembling real-world biological development.

\section{REFERENCES}

[1] W. Arthur. Biased embryos and evolution. Cambridge University Press, 2004.

[2] H. Bart-Smith, K. W. Moored, S. A. Taylor, and T. K. Bliss. Optimization of a tensegrity wing for biomimetic applications. In Proceedings of the 45th IEEE Conference on Decision and Control, pages 2288-2293, 2006.

[3] C. Brangwynne, F. Mackintosh, S. Kumar, N. Geisse, J. Talbot, L. Mahadevan, K. Parker, D. Ingber, and D. Weitz. Microtubules can bear enhanced compressive loads in living cells because of lateral reinforcement. Cell Biology, 173:733-745, 2006.

[4] M. F. Coughlin and D. Stamenović. A tensegrity structure with buckling compression elements: application to cell mechanics. Journal of Applied Mechanics, 64(3):480-486, 1997.

[5] E. H. Davidson. Emerging properties of animal gene regulatory networks. Nature, 468(7326):911-920, 2010.

[6] R. Doursat. Organic Computing, chapter Organically Grown Architectures: creating Decentralized, Autonomous Systems by Embryomorphic Engineering, pages 167-199. Springer, 2008.

[7] M. J. West-Eberhard. Phenotypic plasticity and the origins of diversity. Annual Review of Ecology and Systematics, 20(1):249-278, 1989.

[8] P. Eggenberger. Genome-physics interaction as a new concept to reduce the number of genetic parameters in artificial evolution. In Proceedings of the 5th IEEE Congress on Evolutionary Computation, volume 1, pages 191-198, 2003.

[9] G. Forgács and S. Newman. Biological physics of the developing embryo. Cambridge University Press, 2005.

[10] B. Fuller. Synergetics: explorations in the geometry of thinking. Macmillan, 1975.

[11] B. Goodwin. How the leopard changed its spots: the 
evolution of complexity. Princeton University Press, 2001.

[12] A. Graells Rovira and J. M. Mirats Tur. Control and simulation of a tensegrity-based mobile robot. Robotics and Autonomous Systems, 57(5):526-535, 2009.

[13] J. Henderson and D. Carter. Mechanical induction in limb morphogenesis: the role of growth-generated strains and pressures. Bone, 31(6):645-653, 2002.

[14] G. S. Hornby, H. Lipson, and J. B. Pollack. Generative representations for the automated design of modular physical robots. IEEE Transactions on Robotics and Automation, 19(4):703-719, 2003.

[15] D. Ingber. Cellular tensegrity: defining new rules of biological design that govern the cytoskeleton. Journal of Cell Science, 104(3):613-627, 1993.

[16] D. Ingber. Mechanical control of tissue morphogenesis during embryological development. Developmental Biology, 50:255-266, 2006.

[17] D. Ingber. Cellular mechanotransduction: putting all the pieces together again. The FASEB Journal: Official Publication of the Federation of American Societies for Exp. Biology, 20(7):811-827, 2006.

[18] S. Juan and J. Miratstur. Tensegrity frameworks: static analysis review. Mechanism and Machine Theory, 43(7):859-881, 2008.

[19] S. A. Kauffman. The Origins of Order: self-Organization and Selection in Evolution. Oxford University Press, USA, 1993.

[20] M. Komosinski and A. Rotaru-Varga. Comparison of different genotype encodings for simulated 3D agents. Artificial Life, 7(4):395-418, 2001.

[21] T. Kowaliw and W. Banzhaf. Augmenting artificial development with local fitness. In Proceedings of the 2009 IEEE Congress on Evolutionary Computation, pages 316-323, 2009.

[22] D. Lobo. Evolutionary development based on genetic regulatory models for behavior-finding. $\mathrm{PhD}$ thesis, Universidad de Malaga, 2010.

[23] D. Lobo and F. J. Vico. Evolutionary development of tensegrity structures. Biosystems, 101(3):167-176, 2010 .

[24] T. Mammoto and D. E. Ingber. Mechanical control of tissue and organ development. Development, 137(9):1407-1420, 2010.

[25] R. Motro. Tensegrity: structural systems for the future. Butterworth-Heinemann, 2003.

[26] J. Muñoz, V. Conte, and M. Miodownik. Stress-dependent morphogenesis: continuum mechanics and truss systems. Biomechanics and Modeling in Mechanobiology, 9(4):451-467, 2010.

[27] G. Müller and S. Newman, editors. Origination of organismal form: beyond the gene in developmental and evolutionary biology. The Vienna series in theoretical biology. MIT Press, 2003.

[28] C. Nelson, R. Jean, J. Tan, W. Liu, N. Sniadecki, A. Spector, and C. Chen. Emergent patterns of growth controlled by multicellular form and mechanics.
Proceedings of the National Academy of Sciences of the United States of America, 102:11594-11599, 2005.

[29] S. Newman. Origination of organismal form: beyond the gene in developmental and evolutionary biology, chapter From physics to development: the evolution of morphogenetic mechanisms, pages 221-240. In Muller and Newman [27], 2003.

[30] G. Odell, G. Oster, P. Alberch, and B. Burnside. The mechanical basis of morphogenesis: epithelial folding and invagination. Developmental Biology, 85(2):446-462, 1981.

[31] C. Paul, V. F. Cuevas, and H. Lipson. Design and control of tensegrity robots for locomotion. IEEE Transactions on Robotics, 22(5):944-957, 2006.

[32] J. A. Rieffel, F. J. Valero-Cuevas, and H. Lipson. Morphological communication: exploiting coupled dynamics in a complex mechanical structure to achieve locomotion. Journal of The Royal Society Interface, 7(45):613-621, 2010.

[33] T. Savin, N. A. Kurpios, A. E. Shyer, P. Florescu, H. Liang, L. Mahadevan, and C. J. Tabin. On the growth and form of the gut. Nature, 476(7358):57-62, 2011.

[34] K. Snelson. Continuous tension, discontinuous compression structure (US patent 3169611), 1965.

[35] K. Stanley and R. Miikkulainen. A taxonomy for artificial embryogeny. Artificial Life, 9(2):93-130, 2003.

[36] G. Struhl. A homoeotic mutation transforming leg to antenna in Drosophila. Nature, 292(5824):635-638, 1981.

[37] R. Thom. Structural stability and morphogenesis. Addison Wesley Publishing Company, 1989.

[38] C. H. Waddington. Genetic assimilation of the Bithorax phenotype. Evolution, 1:1-13, 1956.

\section{APPENDIX}

The initial structure contains two concentric rings of 26 equally spaced vertices of mass $m=1$. The radius of the inner ring is 61.5107152186897, and the outer ring 72.1497004210614. All elastic links have a stiffness of $k=20$ and the following actual and resting lengths:

\begin{tabular}{|c|c|c|}
\hline link & $d$ & $l$ \\
\hline \hline$H_{1}$ & 14.8285948251829 & 13.975 \\
\hline$H_{2}$ & 17.3933707403416 & 20.15 \\
\hline$D_{1}, D_{2}$ & 19.2641442449179 & 18.1264008079376 \\
\hline$V_{1}, V_{2}$ & 10.6389852023717 & 11.0406139435269 \\
\hline
\end{tabular}

The exact gene values in the initial genome are: $\left(1,26,\left\{H_{1}\right\}, R, 2.14494510765828\right)$

$\left(1,1,\left\{H_{2}, D_{1}\right\}, K, 0.0\right)$

$\left(2,2,\left\{H_{2}, D_{2}\right\}, K, 0.0\right)$

The simulation was carried out in floating arithmetic with double precision, using the Dorman-Prince method (integrator ode45 in MATLAB ${ }^{\mathrm{TM}}$ ). 